\title{
A Probabilistic Approach to Compound Noun Indexing in Korean Texts
}

\author{
Hyouk R. Park and Young S. Han and Kang H. Lee \\ Korea R\&D Information Center/KIST \\ P.O. Box 122 YuSong Taejon, 305-600, Korea \\ $\{$ hrpark,yshan,khlee\}@stissbs.kordic.re.kr \\ Key-Sun Choi \\ Computer Science Department KAIST \\ YuSong Taejon, 305-701, Korea \\ kschoi@world.kaist.ac.kr
}

\begin{abstract}
In this paper we address the problem of compound noun indexing that is about segmenting or decomposing compound nouns into promising index terms. Compound nouns as index terms that usually subscribe to specific notions tend to increase the precision of retrieval performance. The use of the component nouns of a compound noun as index terms, on the other hand, may improve the recall performance, but can decrease the precision.

Our proposed method to handle compound nouns with a goal to increase the recall while preserving the precision computes the relevance of the component nouns of a compound noun to the document content by comparing the document sets that are supported by the component nouns and the terms of the document. The operational content of a term is represented as the probabilistic distribution of the term over the document set.

Experiments with a set of 1,000 documents show that our method gains $33 \%$ increase of retrieval performance compared to the indexing method without compound noun analysis, and is as good as manual decomposition by human experts.
\end{abstract}

\section{Introduction}

Automatic indexing renders a form of document representation that visualizes the content of the document more explicitly. Indices that are carefully chosen to represent a document will bring about the improvement of retrieval performance in accuracy and time efficiency. The potential of a candidate index is often judged on the basis of its discriminating power over a document set as well as its linguistic significance in the document. Thus, a good index term should distinguish a certain class of documents from the rest of the documents and be relevant to the subject matters of the class of documents to be indexed by the term.

In general, autornatic indexing consists of the identification of index terms and the assignment of weights to the terms (Salton 1983).

An index term can be either a simple noun or a compound noun composed of more than one simple nouns. Compound nouns tend to carry more specific contextual information than simple nouns, thus they are likely to contribute to the retrieval precision. Compound nouns may contain useful simple nouns that usually refer general contexts, and thus will boost the recall of retrieval. Processing compound nouns is decomposing them into simple nouns and evaluating the simple nouns as potential index terms. In both identifying and evaluating index terms, compound nouns require a different strategy from that for simple nouns. 'The identification of compound nouns involves a certain degree of linguistic or statistical analysis that varies from simple stemming to morphological analysis (Fagan 1989).

What makes it even more complicated to handle compound nouns in Korean documents lies in the convention of writing compound nouns. In Korean, it is allowed to write compound nouns with or without intervening blanks between constituent nouns. Arbitrarily long compound nouns are possible and not rare in real texts. The decomposition of a compound noun is particularly problematic because of the severe ambiguity of segmentations.

In this paper, we propose a method to identify and evaluate the candidate index terms from compound nouns. First, each possible decomposition of a compound noun is identified. To' see the potential of the component nouns of the decomposition, we observe how the component nouns are distributed over the total document set, and 
also examine how the simple and compound nouns of the current document are distributed over the same document set. The similarity of the two distributions implies how consistently the two term sets will behave given a query at retrieval time.

The proposed method assumes a dictionary of nouns that is automatically constructed from the document set. This is the practice that has never been tried in Korean document indexing, but has some important merits. A laborious work for the manual construction of nominal dictionaries is not needed. Since the noun dictionary contains only those in a document set, the ambiguity in analyzing words is greatly reduced.

Previous researches on the problem of com pound noun indexing in Korean have been done in two directions. One approach adopts a full-scale morphological analysis to decompose a word into a sequence of the smallest morpheme units that are all treated as index terms. The other approach tries to avoid the complexity of the full scale analysis by using bigrams as in (Fujii 1993; I Lee 1996; Ogawa 1993). Since these methods take all the components of compound nouns as index terms without evaluation, irrelevant terms can decrease retrieval precision.

Experiments on 1000 documents show that our evaluation scheme gave results closer to the luman intuition and maintained the highest preci sion ratio of the existing methods.

In the following section, a brief review of related work on automatic indexing for Korean documents is made. Section 3 explains the proposed method in detail. The verification of the method through experiments is described in section 4 . Section 5 concludes the paper.

\section{Related Work}

The previous approaches to compound noun indexing are based either on full scale morphological analysis (Kang 1995; Kim 1983; Lee 1995; Seo 1993) or on the syllabic patterns (Fujii 1993 ; Lee 1996; Ogawa 1993). Morphological analysis will return morphologically valid component words constituting a given compound word. Since this method does not exclude invalid or meaningless words, it can result in the degradation of precision. Besides the employment of full morphological analysis is often too expensive and requires costly maintenance.

Simpler methods segment compound nouns mechanically into unigram or bigram words that are all regarded as index terms (Iee 1996). Bigram indexes shows better precision than unigrams, but can suffer from big index size. In general, the existing methods for compound noun analysis have been focused mainly on recall performance with little attention to the precision. The work presented in this paper tries to achieve the improvement of recall without the deterioration of preci-

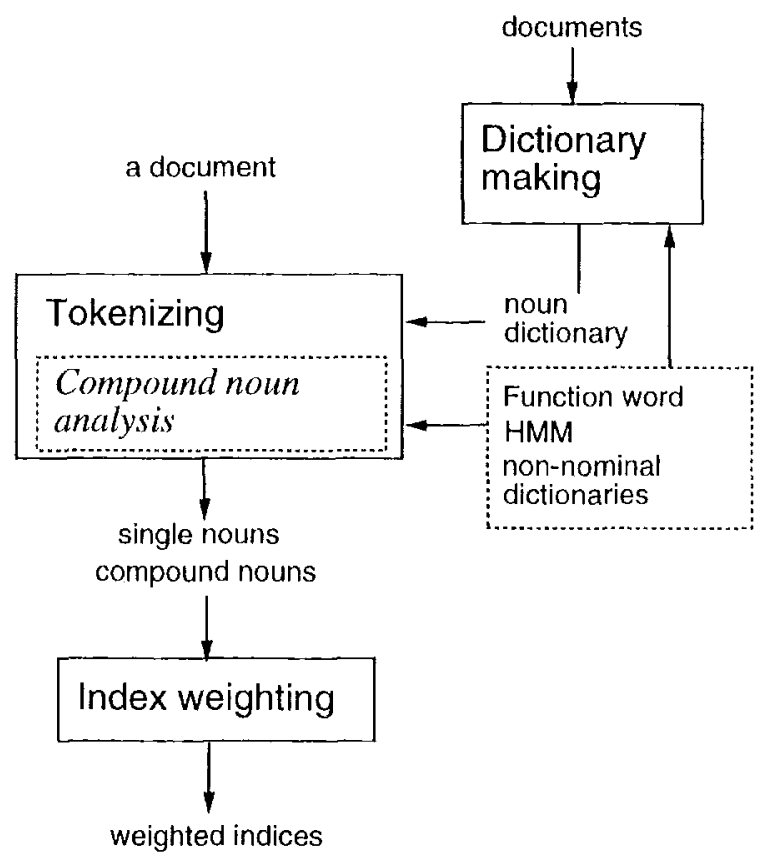

Figure 1: Compound noun indexing.

sion.

\section{Probabilistic Compound Noun Indexing}

In this section, we describe the algorithm to recognize and evaluate candidate index terms from compound nouns. Figure 1 summarizes the algorithm. 'The tokenizer produces a list of simple and compound nouns by utilizing the noun dictionary and the basic stemming rules. 'The noun dictionary is used to identify whether a noun is simple or compound, and the basic stemming rules are used to differentiate nominal words from others such as function words and verbs. The noun dictionary is automatically constructed from the obser vation on the document set. 'The compound noun analyzer investigates if the components of compound nouns are appropriate as indexes. 'The index terms that include simple nouns produced as a result of compound noun analysis are weighted, which finishes the indexing.

let $S$ and $C$ denote the sets of simple and com pound nouns, respectively. Simple nouns are, by definition, those that do not have any of their substrings as a noun according to the dictionary. Compound nouns are those one or more substrings of which are recognized as nouns. Let $T=\left\{T_{1}, T_{2}, \ldots, T_{t}\right\}=S \cup C$ be the set of all simple and compound nouns of a document set. $\Lambda$ lso, let $D=\left\{D_{1}, D_{2}, \ldots, D_{d}\right\}$ be the set of all documents. A docurnent is represented as a list of term-weight $\left(I_{i}, W_{i}\right)$ pairs.

For a compound noun $C_{i}$ of a document, a de- 
composition is a sequence of nouns $\left(T_{1} T_{2} \ldots T_{k}\right)$. In many cases, there are more than one decomposition, but only a fow of them are sensible with respect to the context of the document. Indiscrect use of the component nouns may bring about the improvement of reall, but can lead to the significant decrease of precision. In the following discussions, we describe the details of the algorithm to select useful component nouns from compound nouns.

\subsection{Dictionary buildup}

It is very difficult to provide an IR systiem with the sufficient list of nouns. Because the nominals outnumber and grow faster than other categories of words, it is more efficient to handle non-nominal words manually. Wo consider build. ing noun dictionary by identifying the remaining string as a nom after eliminating non-nominal part of a word. 'The non-nominals are verbs, adverbs, adjectives, prefixes, and suffixes.

'T'he words in non-nominal dictionaries do not include those that can also be used as nouns, which is not a problem since unlike in Finglish, the multi-categorial words in Korean tend to be invariant of meaning. 'The non-nominal dictionaries are made usually by manual work.

'Those recognized as non-nominal words but not as function words are regarded as nouns. There can be multiple interpretations in segmenting a word due to the ambiguity of function words as illustrated in the following example.

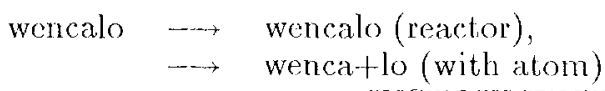

One way to deal with the problem is to use the probability of cach function word and choose the one with the highest value. More accurate measure would be made using a Hidden Markov Model that is about a stochastic process of function words. 'The function words are classified into 32 groups according to their roles and position in sentences. In particular, cach segmentation of a word is evaluated as follows.

$$
P\left(C_{i} \mid C_{i-1}\right) P(n) P(f \mid n)
$$

$P\left(C_{i} \mid C_{i-1}\right)$ is the probability of the function category of current word given the category of the previous word. $P(n)$ is the probability of candidate noun and $P(f \mid n)$ is the probability of a finction word given the candidate noun. 'The best scquence of these segmentations for a sentence can be obtained. The candidate nouns $n$ of the best sequence are then added to the noun dictionary.

\subsection{Tokenizing and compound noun analysis}

'Tokenizing aims at rocognizing simple and compound nouns from a text and reporting them as the the final index terms. 'The method for dictionary making is also used for tokenizing. Since tho dictionary making method gives a list of candidate nouns, we only need to check if a candidate is a componnd noun and judge if the components of the candidate compound noun are consistent with the content of the document.

To deal with the notion of consistency, we have to define the meaning of a term or a set of terms. It is a well recognized practico to regard the discriminating power of a term as the value of the term. 'The quality of the discriminating power is the distribution of the term over a document set. We define the distribution of a term as the mean. ing of the term. Similarly the meaning of a set of terms is the distribution of terms on the document set.

let $M$ be the distribution of a term ' $T_{i}$ over a document set $D=D_{1} \cdots D_{n}$ such that

$$
\sum_{j}^{n} M\left(I_{i}^{\prime}, I_{j}\right)=1 .
$$

One definition of $M(\cdot)$ may be as follows.

$$
M\left(T_{i}, D_{j}\right)=\frac{\operatorname{freq}\left(T_{i}, D_{j}\right)}{\sum_{k} \operatorname{freq}\left(T_{i}, D_{k}\right)} .
$$

For the case of multiple terms,

$$
M\left(\left\{T_{1} \cdots T_{i}\right\}, D_{j}\right)=\frac{\sum_{k=1}^{i} M\left(T_{k}, D_{j}\right)}{i} .
$$

The similarity between two terms (or sets of terms) can be defined as any of vector similarity measurcs. The measurement of rolative information of the two distributions comesponding to the two terms gives the distance between the distributions. Given two distributions $M_{i}$ and $M_{j}$ for $T_{i}$ and $T_{j}$ respectively, the discrimination $T_{0}()$ is defined as follows (Blahut, 1988).

$$
L\left(M_{i}, M_{j}\right)=\sum_{i=0}^{I \cdots L} M_{i} \log \frac{M_{i}}{M_{j}} .
$$

Since wo want the dissimilarity between two distributions, divergence that is a symmetric version of discrimination is more appropriate for our casc. It is defined as follows (Blahut, 1988).

$$
\widetilde{L}\left(M_{i}, M_{j}\right)=L\left(M_{i}, M_{j}\right)+L_{(}\left(M_{j}, M_{i}\right) .
$$

Figure 2 illustrates the different distributions of terms over the same docmrent set suggesting the uscfulness of the distributions as the representiation of the terms. 'The divergence $\bar{r}(\cdot)$ gives about the information (uncertainty) of the two distributions as compared with cach other, and has the following characteristics.

- 'The more uniform the distribution is, the larger $\bar{L}(\cdot)$ will be.

- The morc the two distributions agree, the less $\bar{L}(\cdot)$ will be. 


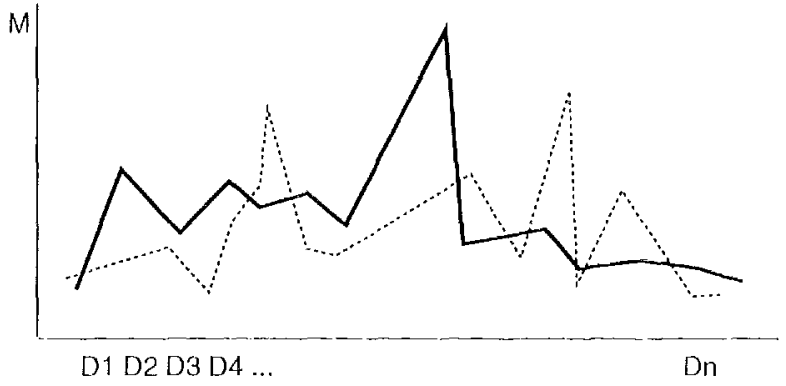

Jigure 2: Illustration of term distributions over the same document set

The characteristics are uschul because good index terms should be less uniform and share similar contexts with other terms in a docmment. In this respect, information theoretic measure is more concrete and thus possibly more accurate than vector similarity measures.

lor each decomposition $\left(T_{i}, \cdots, T_{j}\right)$ of a com pound noun $C_{k}$, what, we want to see is loow different the decomposed terms and the docmonent terms are. 'That is, $T\left(\left\{T_{i}, \cdots, T_{j}\right\}, D_{k}\right)$ becomes the score of the particalar decomposition. What we select here is one decomposition with the low est divergence. Letting $\tau$ and $\tau$ ' denote a decomposition and the best decomposition respectively,

$$
\tau^{\prime}=\underset{\tau}{\arg \min } \bar{T}\left(\tau, I_{k}\right)
$$

'The following summarizes the procedure of extracting simple nouns from conpound nouns.

1. Remove non-nominal words msing the method for dictionary making.

2. Identify compound nouns using nominal dictionary.

3. For cach decomposition $\tau_{i}$ of a compound noun $C_{i}^{*}$, compule $\bar{l}\left(\tau_{i}, D\right)$.

4. Select $\tau_{i}$ with the lowest $\left.\bar{l}\left(\tau_{i}, l\right)\right)$.

\subsection{Index weighting}

'There are three well known nethods for weighting index terms. They are based on the information of inverse document frecfuency, discrimina tion value, and probabilistic value (Salton 1988). It turned out that these methods lead to similar performance, but inverse document frequency is by far the simplest of them in terms of time complexity and required resonecs (Salton 1988; Harmann 1992).

Tuverse document frequency method is also shown to work with litte performance variation across different somatins. fior this reason, wo adopted inverse document frequency in the experiments. It is defined as follows.

$$
w_{i j}=t f_{i j} \times \log \left(\frac{1}{d f_{i}}\right)
$$

Table 1: The proportion of compound nomms in the 1000 science aldstract. About $9 \%$ of nombs are compound nouns.

\begin{tabular}{rrr} 
no. of components & nomms & proportion \\
\hline 1 & 49639 & $90.55 \%$ \\
2 & 1665 & $8.50 \%$ \\
3 & 469 & $.85 \%$ \\
4 & 53 & $.09 \%$ \\
5 & 6 & $.01 \%$
\end{tabular}

where $w_{i j}$ is the weight of the i'th term in the f'th docament, $t_{i j}$ is the mumber of occurrencess of the ith term in the j'th document, and $d f_{i}$ is the mumber of clocuments in which the ith term occurs

\section{Fxperimentis}

The goal of experiments is to validate the proposed algorithm for analyzing compound noms by comparing it with the manual analysis and the bigram mothod.

"The test data set consists of 1000 science al) stracts written in Korean (Kin 1994). All noninals are thantally identified and compound nomes were decomposed into appropriate simple nouns by an expert inclexer. In the linst experinent, our proposed algorithen is asked to do the same thing over the test data, and retrieval perfor mances on the two different ontconess (manually indexed and antomatically indexed abstracts) are compared. In the second experintents, the performances of the proposed method and bigram method are compared to observe how the precision is affected.

As is shown at table 1 , the portion of compound nonns is abont $9 \%$ of total nouns found in the test set, but can make critical eflects on the retricval performance because often compound nonns carrying more specific information become a more anculate index to the documents.

Figure 3 and lable 2 sumnarize the perlormance of the indexing methods: manual analy. sis, the proposed probabilistic method, and the bigran method. 'The proposed method showed a slightly better performance (around $3 \%-4 \%$ ) than manual indexing or bigram indexing. However, our method has was more efficient than bigram indexing in terms of the number of index terms and the average number of retrieved documents per a query.

The average ambiguity of a compound noun is 1.43 , and this low ambiguity must have contributed to the high agreement ratio of the proposed indexing method with manual indexing. 'The low ambiguity is partly attributed to the noun dictionary that has no unnecessary cntrics 


\begin{tabular}{|l|l|l|l|l|}
\hline Recall & Man. & Prob. & Big. & No Anal. \\
\hline 0.00 & 0.8719 & 0.8579 & 0.8406 & 0.7957 \\
0.10 & 0.7719 & 0.7587 & 0.7841 & 0.6455 \\
0.20 & 0.7122 & 0.6981 & 0.6812 & 0.5894 \\
0.30 & 0.5895 & 0.6312 & 0.5939 & 0.4931 \\
0.40 & 0.5458 & 0.5854 & 0.5637 & 0.4103 \\
0.50 & 0.4957 & 0.5287 & 0.5240 & 0.3646 \\
0.60 & 0.4272 & 0.4438 & 0.4370 & 0.2844 \\
0.70 & 0.3304 & 0.3665 & 0.3322 & 0.2311 \\
0.80 & 0.2552 & 0.2876 & 0.2569 & 0.1695 \\
0.90 & 0.2102 & 0.2280 & 0.2028 & 0.0900 \\
1.00 & 0.1428 & 0.1724 & 0.1600 & 0.0514 \\
\hline
\end{tabular}

Table 2: Performance of Manual, Prob., and Bigram Indexing

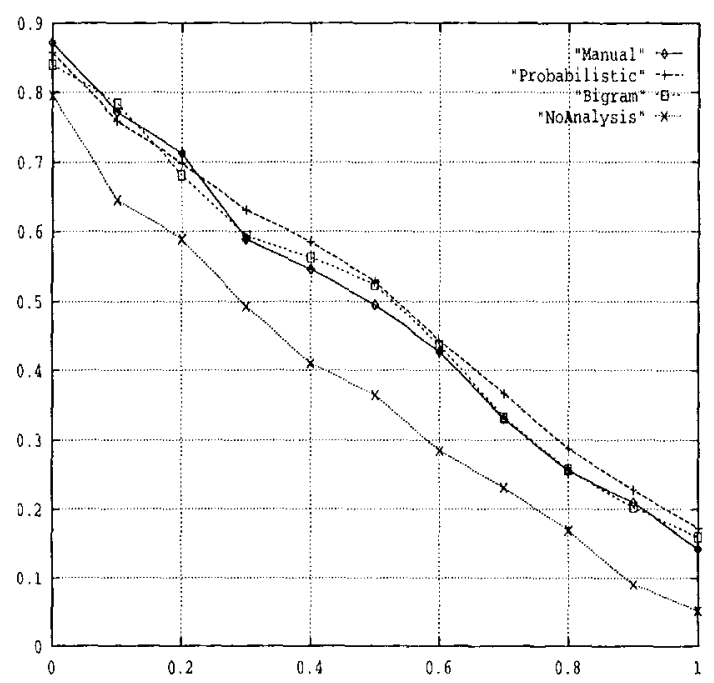

Figure 3: Recall-Precison curve of indexing methods

not found at the documents.

\section{Conclusion}

The compound analysis in automatic indexing aims at the improvement of recall performance by extracting useful component nouns from compound nouns. The task for Korean texts requires extra efforts due to the complexity of inflections. The proposed method gives better potential of sustaining the precision while improving the recall than other approaches by making use of probabilistic distributions of terms as the representation of meaning of the terms.

The proposed method to evaluate the components of compound nouns is unique in that it defines and uses term representation, which explains the superiority of the method to other methods. The method requires little human involvement and is very promising for the implementation of practical systems by achieving efficiency and accuracy at the same time.

\section{References}

Blahut, Richard E. (1987). Principles and Practice of Information Theory. Addison-Wesley.

Fagan, J. L. (1989). The effectiveness of a Nonsyntactic Approach to Automatic Phrase Indexing for Document Retrieval, Journal of American Society for Information Science, Vol. 40, No. 2.

IIarman, D. (1992). "Ranking Algorithms" in Information Retrieval: Data Structure and Algorithms, (Frakes, W. B., and Baeza-Yates, R. ed.) Prentice Hall.

Fujii, H., and Croft, W. B. (1993). "A comparison of indexing techniques for Japanese text retrieval," In Proceedings of 16'th ACM SIGIR Conference.

Kang, S. S. (1995). "Role of Morphological Analysis for Korean Automatic Indexing,", In Proceedings of the 22 nd Korea Information Science Society Conference.

Kim, Y. H. (1983). Automatic Indexing System of Korean Texts mixed with Chinese and English M.S. Thesis, Dept. of Computer Science, Korea Advanced Institute of Science and Technology.

Kim, S. H. (1994). A Development of the 'Test Collection for Estimating the Retrieval Performance of an Automatic Indexer, Journal of Korea Information Management Society, Vol. 11, No. 1.

Lee, J. H. (1996). "n-Gram-Based Indexing for Effective Retrieval of Korean Texts," In Proceedings of 1st Australian Document Computing Symposium 1996

Lee, H. A. (1995). "Implementation of an Indexing System Based on Korean Morpheme Structural Rules,", In Proceedings of Spring Conference of Korea Information Science Society.

Ogawa Y. (1993). "Simple word strings as compound keywords: An indexing and ranking method for Japancse texts,", In Proceedings of 16 'th ACM SIGIR Conference.

Salton, G., and McGill M. J. (1983). Introduction to Modern Information Retrieval McGraw-Hill Inc:

Salton, G., and Buckey, C. (1988). Term Weighting Approaches in Automatic Text Retrieval, Information Processing 6 and Management, Vol. 24, No. 5 .

Seo, F. K. (1993). An Experiment in Automatic Indexing with Korean Texts: A Comparison of Syntactico-Statistical and Manual Methods, Journal of Korea Information Management Society, Vol. 10, No. 1. 